\title{
Principal Ideals in Matrix Rings
}

\author{
Morris Newman and Stephen Pierce \\ Institute for Basic Standards, National Bureau of Standards, \\ Washington, D.C. 20234
}

(April 21, 1969)

\begin{abstract}
It is shown that every left ideal of the complete matrix ring of a given order over a principal ideal ring is principal, and a partial converse is proven.

Key words: Dedekind ring; matrix ring; non-Noetherian ring; principal ideal ring.
\end{abstract}

\section{Introduction}

Let $R$ be a ring with a unity 1 , and let $n$ be a positive integer. It is well-known [3, p. 37 ${ }^{1}$ that every two-sided ideal of $R_{n}$ (the complete matrix ring of order $n$ over $R$ ) is necessarily of the form $M_{n}$, where $M$ is a two-sided ideal of $R$. Simple examples show that this result no longer holds for one-sided ideals. In this note we investigate the left ideals of $R_{n}$ in the case when $R$ is a principal ideal ring (an integral domain in which every ideal is principal). We shall prove

THEOREM 1: If $\mathrm{R}$ is a principal ideal ring, then every left ideal of $\mathrm{R}_{\mathrm{n}}$ is principal.

The proof of Theorem 1 depends upon the fact that if $A$ is any $p \times q$ matrix over $R$, then a unit matrix $U$ of $R_{p}$ exists such that the $p \times q$ matrix $U A$ is upper triangular [2, p. 32].

We also establish the following partial converse to Theorem 1:

Theorem 2: If $\mathrm{R}$ is not Noetherian or if $\mathrm{R}$ is a Dedekind ring but not a principal ideal ring, then $\mathrm{R}_{\mathrm{n}}$ contains a nonprincipal left ideal.

For general information on rings, see [3]. For information on Dedekind rings, see [1, p. 101].

\section{Proofs}

We denote the matrix of $R_{n}$ which has 1 in position $(i, j)$ and 0 elsewhere by $E_{i j}$. We first prove of $R_{n}$.

LEMma 1: Suppose that every left ideal of $\mathrm{R}$ has a finite R-basis. Then so has every left ideal

ProOF: Let $a$ be a left ideal of $R_{n}$. Let $a_{k}, 2 \leqslant k \leqslant n$, be the subset of $a$ consisting of all matrices of $a$ whose first $k-1$ columns are 0 ; and set $a_{1}=a$. Then, as is easily verified, $a_{k}$ is a left ideal of $R_{n}$ for $1 \leqslant k \leqslant n$.

Let $M_{i k}, 1 \leqslant i \leqslant n$, be the set of elements of $R$ occurring in the $(i, k)$ position of all matrices of $a_{k}, 1 \leqslant \mathrm{k} \leqslant \mathrm{n}$. Then $M_{i k}$ is a left ideal of $R$ (since $a_{k}$ is a left ideal of $R_{n}$ ) and so has a finite $R$-basis, say

$$
m_{i k}^{l}, 1 \leqslant l \leqslant l_{i k} .
$$

Hence we can find $l_{i k}$ matrices of $a_{k}$, say $A_{i k}^{l}$, such that the $(i, k)$ th entry of $A_{i k}^{l}$ is $m_{i k}^{l}$ It follows that the $l_{i k}$ matrices

$$
B_{i k}^{l}=E_{i i} A_{i k}, 1 \leqslant i, k \leqslant n, 1 \leqslant l \leqslant l_{i k},
$$

also belong to $a_{k}$, have $m_{i k}^{l}$ as their $(i, k)$ th entry, but have nonzero entries in the $i$ th row only. These

\footnotetext{
${ }^{1}$ Figures in brackets indicate the literature at the end of this paper.
} 
matrices constitute a finite $R$-basis for $a$. For suppose that $A$ is any element of $a$. We first find elements $r_{i 1}^{l}$ of $R$ such that

$$
A-\sum_{i=1}^{n} \sum_{l=1}^{l_{i 1}} r_{i 1}^{l} B_{i 1}^{l}=A_{2} \epsilon a_{2}
$$

we then find elements $r_{i 2}^{l}$ of $R$ such that

$$
A_{2}-\sum_{i=1}^{n} \sum_{l=1}^{l_{i 2}} r_{i 2}^{l} B_{i 2}^{l}=A_{3} \epsilon a_{3}
$$

and continuing in this manner, we determine elements $r_{i k}^{l}$ of $R$ such that

$$
A=\sum_{k=1}^{n} \sum_{i=1}^{n} \sum_{l=1}^{l_{i k}} r_{l k}^{l} B_{i k}^{l}
$$

This completes the proof.

We now prove Theorem 1. Let $a$ be a left ideal of $R_{n}$. By Lemma 1, $a$ possesses a finite $R$-basis, say $B_{1}, B_{2}, \ldots, B_{t}$. Let $B$ be the $n t \times t$ matrix

$$
B=\left(\begin{array}{c}
B_{1} \\
B_{2} \\
\cdot \\
\cdot \\
\cdot \\
B_{t}
\end{array}\right)
$$

Let $U$ be a unit matrix of $R_{n t}$ such that $U B=T$ is upper triangular. Thus

$$
U B=T=\left[\begin{array}{c}
H \\
0
\end{array}\right],
$$

where $H$ is an $n \times n$ upper triangular matrix, and the zero block 0 is $(n t-n) \times n$. We shall show that $a=R_{n} H$. For if we write $U=\left(U_{i j}\right)$, where the matrices $U_{i j}$ are $n \times n$, then

so that $H \epsilon a$, implying that

$$
\begin{gathered}
\sum_{j=1}^{t} U_{1 j} B_{j}=H, \\
R_{n} H \subset a .
\end{gathered}
$$

If we then write $U^{-1}=V=\left(V_{i j}\right)$, where the matrices $V_{i j}$ are $n \times n$, then $V$ belongs to $R_{n t}$ (since $U$ is a unit matrix of $R_{n t}$ ) and from $B=V T$ we find that

implying that

$$
\begin{gathered}
B_{i}=V_{i 1} H, \quad 1 \leqslant i \leqslant t, \\
a \subset R_{n} H .
\end{gathered}
$$

This completes the proof of Theorem 1.

To prove Theorem 2, we first observe that for any left ideal $M$ of $R$, the left ideal $M_{n}$ of $R_{n}$ can be principal only if $M$ has a set of $n$ or fewer generators. In particular, if $R$ is non-Noetherian, $M$ can be chosen to violate this condition.

We now assume that $R$ is a Dedekind ring and that any ideal in $R$ can be generated by at most $n$ elements. Let $S$ be a nonprincial ideal in $R$, and let $\mathscr{S}$ be the subset of $R_{n}$ consisting of all matrices with first column entries in $S$ and all other entries arbitrary members of $R$. Clearly, $\mathscr{S}$ is a left ideal in $R_{n}$. We shall show that $\mathscr{S}$ is not principal.

Suppose the contrary. Let $X=\left(x_{i j}\right)$ generate $\mathscr{S}$, so that $\mathscr{S}=R_{n} X$. Clearly the $x_{i 1}$ generate $S ; S=\left\{x_{11}, x_{21}, \ldots, x_{n 1}\right\}$. We may assume that $x_{11}$ is not zero, since we may interchange the rows of $X$ by left multiplication by a permutation matrix. Let $d=\operatorname{det} X$. Since $\mathscr{S}$ contains nonsingular matrices (for example, $\left.\operatorname{diag}\left(x_{11}, 1, \ldots, 1\right)\right) X$ must be nonsingular and thus $d$ is a nonzero element of $S$. Let $Y=\left(y_{i j}\right)$ be the adjoint of $X$, so that

$$
X Y=Y X=d I .
$$


Then $Y \in R_{n}$, and if $C$ is any matrix in $\mathscr{S}$, every element of $C Y$ must be divisible by $d$. First choose $C=x_{i 1} E_{11}, 1 \leqslant i \leqslant n$. We obtain

$$
\{d\} \mid\left\{x_{i 1} y_{l j}\right\}, \quad 1 \leqslant i, j \leqslant n .
$$

Next choose $C=E_{1 j}, 2 \leqslant i \leqslant n$. We obtain

$$
\{d\} \mid\left\{y_{i j}\right\}, \quad 2 \leqslant i \leqslant n, \quad 1 \leqslant j \leqslant n .
$$

Put $y=\left\{y_{11}, y_{12}, \ldots, y_{1 n}\right\}$. Then (2) implies that $y\{d\}^{n-1} \mid\{\operatorname{det} Y\}$; and since $\operatorname{det} Y=d^{n-1}, y=\{1\}=$ $R$. Hence $\left\{y_{11}, y_{12}, \ldots, y_{1 n}\right\}=\{1\}=R$. But now (1) and (2) imply that $\{d\} \mid\left\{x_{i 1}\right\}, 1 \leqslant i \leqslant n$. Write

$$
x_{i 1}=\beta_{i} d, \quad \beta_{i} \in R, \quad 1 \leqslant i \leqslant n .
$$

Since $d \epsilon S$ and the $x_{i 1}$ are a basis for $S$, elements $r_{i}$ of $R$ exist such that

But now (3) implies that

$$
d=\sum_{i=1}^{n} r_{i} x_{i 1}
$$

and hence

$$
\sum_{i=1}^{n} r_{i} \beta_{i}=1
$$

$$
S=\left\{x_{11}, x_{21}, \ldots, x_{n 1}\right\}=\left\{\beta_{1}, \beta_{2}, . ., \beta_{n}\right\}\{d\}=\{d\} .
$$

Thus $S$ is principal, a contradiction. This completes the proof of Theorem 2 .

\section{References}

[1] Curtis, C., and Reiner, I., Representation theory of finite groups and associated algebras, Interscience (1962).

[2] MacDuffee, C. C., The theory of matrices, Chelsea (1946).

[3] McCoy, N. H., The theory of rings, Macmillan (1964).

(Paper 73B3-300) 